\title{
ON THE ISOMETRIC EMBEDDING OF TORUS IN $\mathbb{R}^{3 *}$
}

\author{
QING $\mathrm{HAN}^{\dagger}$ AND FANGHUA LIN ${ }^{\ddagger}$
}

Dedicate to Professor Neil Trudinger with admiration and friendship

Key words. Isometric embedding, rigidity, curvature, equations of mixed type.

AMS subject classifications. 53C24, 53C 42

1. Introduction. In 1916, Weyl [16] posed the following problem: Does any smooth metric on $\mathbb{S}^{2}$ with pointwise positive Gauss curvature admit a smooth isometric embedding in $\mathbb{R}^{3}$ ? The first attempt to solve the problem was made by Weyl himself. He suggested the continuity method and obtained a priori estimates up to the second derivatives. It was after twenty years that Lewy [10] solved the problem in the case of $g$ being analytic. The smooth case of the Weyl problem was solved independently by Nirenberg [13] and Pogorelov [15]. Nirenberg used the continuity method and provided a solution if the metric $g$ is smooth. In a completely different approach to the problem, Alexandroff in [2] obtained a generalized solution of the Weyl problem as a limit of polyhedra. The regularity of this generalized solution was proved by Pogorelov in [15]. Refer to [6] for detailed discussions. We point out that the isometric embedding in $\mathbb{R}^{3}$ of smooth metrics on $\mathbb{S}^{2}$ with positive Gauss curvature remains to be the only result of the isometric embedding of compact surfaces in $\mathbb{R}^{3}$.

In this paper, we initiate a study of the isometric embedding in $\mathbb{R}^{3}$ of smooth metrics on torus. We first start with a general discussion of compact surfaces. Suppose $\left(M^{2}, g\right)$ is a compact surface, a 2-dimensional compact Riemannian manifold without boundary. It is well-known that if $g$ is isometrically embedded in $\mathbb{R}^{3}$ then

$$
\int_{\{K>0\}} K d g \geq 4 \pi
$$

where $K$ is the Gauss curvature of $g$. Equality holds if and only if $\{p ; K(p)>0\}$ is convex in $\mathbb{R}^{3}$. It is unlikely that (1.1) itself is sufficient to yield the isometric embedding of $(M, g)$ in $\mathbb{R}^{3}$.

To find sufficient conditions for the existence of isometric embedding, we shall first examine the rigidity, the uniqueness of the isometric embedding if it exists. The first rigidity result was proved by Cohn-Vossen [3]; this states that any two closed isometric analytic convex surfaces are congruent to each other. Later, Herglotz [8] gave a very short proof of the rigidity, assuming that the surfaces are smooth. For compact surfaces with Gauss curvature of mixed sign, Alexandrov [1] introduced a class of surfaces satisfying some integral condition for its Gauss curvature and proved that any compact analytic surface with this condition is rigid. Then, Nirenberg [14] generalized this result to smooth surfaces. To do this, he needed some extra conditions, one of which is not intrinsic. Again, refer to [6] for detailed discussions.

\footnotetext{
* Received April 6, 2008; accepted for publication June 13, 2008.

$\dagger$ Department of Mathematics, University of Notre Dame, Notre Dame, IN 46556, USA (qhan@ nd.edu); School of Mathematical Sciences, Peking University, Beijing 100871, China. Research of the first author was partially supported by the NSF grant DMS0654261.

${ }^{\ddagger}$ Courant Institute, 251 Mercer Street, New York, NY 10012, USA (linf@cims.nyu.edu); Department of Mathematics, East China Normal University, Shanghai 200062, China. Research of the second author was partially supported by the NSF grant DMS0700517.
} 
Now we briefly recall the main result in [14] because of its importance in our paper. Let $(M, g)$ be a compact 2-dimensional Riemannian manifold without boundary such that

$$
\int_{\{K>0\}} K d g=4 \pi
$$

and

$$
\nabla K \neq 0 \text { whenever } K=0 .
$$

The assumption (1.3) means the Gauss curvature changes sign cleanly and it implies that $\{p \in M ; K(p)=0\}$ consists of finitely many closed curves in $M$. Let $M_{+}=$ $\{p \in M ; K(p)>0\}$. It is proved in [14] that $\left(M_{+},\left.g\right|_{M_{+}}\right)$is rigid in $\mathbb{R}^{3}$ and that $(M, g)$ is rigid if there is at most one closed asymptotic curves in each component of $M_{-}=\{p \in M ; K(p)<0\}$. We need to point out that the extra assumption on asymptotic curves is not intrinsic. With this rigidity result, it seems reasonable to start with 2-dimensional compact Riemannian manifolds $(M, g)$ satisfying (1.2) and (1.3) in our discussion of the isometric embedding of $(M, g)$ in $\mathbb{R}^{3}$.

We now return to torus. Suppose $\left\{\mathbb{T}^{2}, g_{0}\right\}$ is a standard torus with the standard metric in $\mathbb{R}^{3}$. It is easy to check that $\left\{\mathbb{T}^{2}, g_{0}\right\}$ satisfies (1.2) and (1.3). For metrics on $\mathbb{T}^{2}$, instead of (1.3), we assume

$$
\{K=0\} \text { consists of two curves where } \nabla K \neq 0 .
$$

We propose the following problem.

Problem 1.1. Find sufficient conditions besides (1.2) and (1.4) on smooth metrics $g$ on $\mathbb{T}^{2}$ so that $g$ can be isometrically embedded in $\mathbb{R}^{3}$. Furthermore, what is the structure of those metrics in the space of smooth metrics on $\mathbb{T}^{2}$ ?

Let $g$ be a smooth metric on $\mathbb{T}^{2}$ satisfying (1.4). By applying the result of the local isometric embedding with Gauss curvature changing type cleanly obtained by C.-S. Lin [11], we conclude that $g$ can be isometrically embedded in $\mathbb{R}^{3}$ locally at points where the Gauss curvature vanishes. The local isometric embedding for other parts where the Gauss curvature does not vanish is straightforward. The question is whether these small pieces form a global torus-like surface.

To study Problem 1.1, we may first concentrate on special classes of metrics. For example, we may start with metrics on torus which are close to the standard metric. We note that (1.4) holds for any metrics on $\mathbb{T}^{2}$ which are close to the standard metric since it holds for the standard metric. Moreover, the non-intrinsic assumption on asymptotic curves in Niregberg's rigidity result is also satisfied by metrics on the torus which are perturbations of the standard metric. Therefore Nirenberg's theorem asserts that up to a rigid motion there is only one embedding if it exists. Another special class of metrics on torus corresponds to surfaces of revolution in $\mathbb{R}^{3}$. These are the metrics we will study in this paper. As we will see, (1.2) and (1.4) are not sufficient for the isometric embedding of these metrics in $\mathbb{R}^{3}$. An extra condition is needed to ensure that those metrics are realized by closed surfaces. Based on this, we can construct smooth metrics on torus satisfying (1.2) and (1.4) which cannot be realized by torus-like surfaces in $\mathbb{R}^{3}$. In fact, these metrics can be made as perturbations of the standard metric $g_{0}$ and satisfy (1.2). 
To present the main result, we identity $\mathbb{T}^{2}=\mathbb{S}^{1} \times \mathbb{S}^{1}$ and denote $(x, t) \in \mathbb{S}^{1} \times \mathbb{S}^{1}$. Let $g$ be a smooth metric on $\mathbb{T}^{2}$ given by

$$
g=E(t) d x^{2}+G(t) d t^{2},
$$

where $E$ and $G$ are smooth positive $2 \pi$-periodic functions.

The following is the main result in this paper.

TheOREM 1.2. Suppose that $g$ is a metric on $\mathbb{T}^{2}$ as in (1.5) and satisfies (1.2) and (1.4) with $K=0$ on $\left\{t=t_{1}\right\}$ and $\left\{t=t_{2}\right\}$ for some $t_{1}, t_{2} \in[0,2 \pi)$ with $t_{1}<t_{2}$. Then $g$ admits a smooth isometric embedding in $\mathbb{R}^{3}$ if and only if

$$
\int_{t_{1}}^{t_{2}} \sqrt{1-\left(\frac{E^{\prime}}{2 \sqrt{E G}}\right)^{2}} \sqrt{G} d t=\int_{t_{2}}^{t_{1}+2 \pi} \sqrt{1-\left(\frac{E^{\prime}}{2 \sqrt{E G}}\right)^{2}} \sqrt{G} d t .
$$

We will interpret (1.2) and (1.6) for metrics given in (1.5) in the proof of Theorem 1.2 .

As we know, basic equations for the isometric embedding of 2-dimensional Riemannian manifolds in $\mathbb{R}^{3}$ can be expressed either by the Darboux equation, a fully nonlinear equation of Monge-Ampère type, or the Gauss-Codazzi equation, a quasilinear differential system of first order. These equations are of mixed type in our setting, elliptic if the Gauss curvature is positive and hyperbolic if the Gauss curvature is negative. Few results are available for global solutions of differential equations of mixed type.

To prove Theorem 1.2, we first construct an isometric embedding by a surface of revolution and then use the rigidity result by Nirenberg to prove there is no isometric embedding in other forms of surfaces. Details will be given in the next section.

2. Proof of Theorem 1.2. First, we recall a result.

Lemma 2.1. Suppose a 2-dimensional metric $g$ is given by

$$
g=E(x, t) d x^{2}+G(x, t) d t^{2} .
$$

Then its Gauss curvature is

$$
K=-\frac{1}{2 \sqrt{E G}}\left\{\left(\frac{G_{x}}{\sqrt{E G}}\right)_{x}+\left(\frac{E_{t}}{\sqrt{E G}}\right)_{t}\right\} .
$$

We may also write $K$ as

$$
K=-\frac{1}{\sqrt{E G}}\left\{\partial_{x}\left(\frac{\partial_{x} \sqrt{G}}{\sqrt{E}}\right)+\partial_{t}\left(\frac{\partial_{t} \sqrt{E}}{\sqrt{G}}\right)\right\} .
$$

The proof is based on a straightforward calculation. Refer to Lemma 2.1.1 in [6] for details.

Now we focus on surfaces of revolution in $\mathbb{R}^{3}$. We identity $\mathbb{T}^{2}=\mathbb{S}^{1} \times \mathbb{S}^{1}$ and denote $(x, t) \in \mathbb{S}^{1} \times \mathbb{S}^{1}$. Consider a closed curve $\{(0, f(t), h(t))\}$ in $\mathbb{R}^{3}$ with $f(t)>0$. Here $f(t)$ and $h(t)$ are periodic in $t \in[0,2 \pi]$, and $t$ is the angle in the $x_{2}-x_{3}$ plane from the positive $x_{2}$-direction. Denote by $x$ the angle in the $x_{1}-x_{2}$ plane from the positive $x_{2}$-direction. Consider a surface of revolution in $\mathbb{R}^{3}$

$$
\mathbf{r}(x, t)=(f(t) \cos x, f(t) \sin x, h(t)) .
$$


The induced metric on $\mathbf{r}$ has the form

$$
g=f^{2} d x^{2}+\left(f^{\prime 2}+h^{\prime 2}\right) d t^{2} .
$$

For future reference, we note that a unit normal vector is given by

$$
\mathbf{n}(x, t)=\frac{1}{f^{\prime 2}(t)+g^{\prime 2}(t)}\left(h^{\prime}(t) \cos x, h^{\prime}(t) \sin x,-f^{\prime}(t)\right),
$$

and the corresponding second fundamental form is given by

$$
I I=\frac{1}{f^{\prime 2}+g^{\prime 2}}\left(-f h^{\prime} d x^{2}+\left(h^{\prime} f^{\prime \prime}-f^{\prime} h^{\prime \prime}\right) d t^{2}\right) .
$$

We point out that the second fundamental form is diagonal.

A standard torus and its induced metric are given by

$$
f(t)=a+\cos t, \quad h(t)=\sin t,
$$

and

$$
g_{0}=(a+\cos t)^{2} d x^{2}+d t^{2},
$$

for some constant $a>1$.

In the following, we write the metric $g$ in $(2.2)$ by

$$
g=E(t) d x^{2}+G(t) d t^{2},
$$

where $E$ and $G$ are smooth positive $2 \pi$-periodic functions given by

$$
f^{2}=E, \quad f^{\prime 2}+h^{\prime 2}=G .
$$

We take $f=\sqrt{E}$ and then have

$$
{h^{\prime}}^{2}=G-\left(\frac{E^{\prime}}{2 \sqrt{E}}\right)^{2}=G\left(1-\left(\frac{E^{\prime}}{2 \sqrt{E G}}\right)^{2}\right) .
$$

This implies

$$
\left|E^{\prime}\right| \leq 2 \sqrt{E G} .
$$

In the following, we assume that the Gauss curvature $K$ satisfies (1.2), i.e.,

$$
\int_{\{K>0\}} K d g=4 \pi
$$

By Lemma 2.1, we have

$$
K=-\frac{1}{2 \sqrt{E G}}\left(\frac{E^{\prime}}{\sqrt{E G}}\right)^{\prime},
$$

and hence

$$
K d g=-\left(\frac{E^{\prime}}{2 \sqrt{E G}}\right)^{\prime} d x d t .
$$


Now we assume there are $t_{1}, t_{2} \in[0,2 \pi)$ with $t_{1}<t_{2}$ such that

$$
\begin{aligned}
& \left(\frac{E^{\prime}}{2 \sqrt{E G}}\right)^{\prime}>0 \text { on } \Gamma_{-}=\left\{t ; t_{1}<t<t_{2}\right\}, \\
& \left(\frac{E^{\prime}}{2 \sqrt{E G}}\right)^{\prime}<0 \text { on } \Gamma_{+}=\left\{t ; t_{2}<t<t_{1}+2 \pi\right\} .
\end{aligned}
$$

Then we have

$$
K>0 \text { on } \mathbb{S}^{1} \times \Gamma_{+}, \quad K<0 \text { on } \mathbb{S}^{1} \times \Gamma_{-},
$$

and

$$
\begin{aligned}
\int_{\{K>0\}} K d g & =-2 \pi \int_{\left\{t \in \mathbb{S} ;\left(\frac{E^{\prime}}{2 \sqrt{E G}}\right)^{\prime}<0\right\}}\left(\frac{E^{\prime}}{2 \sqrt{E G}}\right)^{\prime} d t \\
& =2 \pi\left(\frac{E^{\prime}}{2 \sqrt{E G}}\left(t_{1}\right)-\frac{E^{\prime}}{2 \sqrt{E G}}\left(t_{2}\right)\right) .
\end{aligned}
$$

Hence, (1.2) is equivalent to

$$
\frac{E^{\prime}}{\sqrt{E G}}\left(t_{1}\right)-\frac{E^{\prime}}{\sqrt{E G}}\left(t_{2}\right)=4
$$

Note that (2.5) implies

$$
\begin{aligned}
& \frac{E^{\prime}}{\sqrt{E G}} \text { has only two critical points } t_{1} \text { and } t_{2} \text { on } \mathbb{S}^{1} \text {, and } \\
& \frac{E^{\prime}}{\sqrt{E G}} \text { attains its maximum at } t_{1} \text { and its minimum at } t_{2} .
\end{aligned}
$$

With (2.4), we get

$$
\frac{E^{\prime}}{\sqrt{E G}}\left(t_{1}\right)=2, \quad \frac{E^{\prime}}{\sqrt{E G}}\left(t_{2}\right)=-2 .
$$

Last, we note that $h^{\prime}$ is a function of mixed sign on $\mathbb{S}^{1}$ with $h^{\prime}\left(t_{1}\right)=h^{\prime}\left(t_{2}\right)=0$. Hence,

$$
h^{\prime}(t)= \pm \sqrt{G-\left(\frac{E^{\prime}}{2 \sqrt{E}}\right)^{2}} \text { on } \Gamma_{ \pm},
$$

or

$$
h^{\prime}(t)=\mp \sqrt{G-\left(\frac{E^{\prime}}{2 \sqrt{E}}\right)^{2}} \text { on } \Gamma_{ \pm} .
$$

With $\int_{0}^{2 \pi} h^{\prime}(t) d t=0$, we have

$$
\int_{t_{1}}^{t_{2}} \sqrt{G-\left(\frac{E^{\prime}}{2 \sqrt{E}}\right)^{2}}=\int_{t_{2}}^{t_{1}+2 \pi} \sqrt{G-\left(\frac{E^{\prime}}{2 \sqrt{E}}\right)^{2}} .
$$


The following result characterizes whether metrics in (1.5) can be realized by surfaces of revolution.

Lemma 2.2. Suppose that $g$ is a metric on $\mathbb{T}^{2}$ as in (1.5) such that

$$
\begin{gathered}
\left|\frac{E^{\prime}}{\sqrt{E G}}\right| \leq 2 \text { and } \frac{E^{\prime}}{\sqrt{E G}} \text { has only two critical points } t_{1} \text { and } t_{2} \text { with } \\
\frac{E^{\prime}}{\sqrt{E G}}\left(t_{1}\right)=2, \quad \frac{E^{\prime}}{\sqrt{E G}}\left(t_{2}\right)=-2 .
\end{gathered}
$$

Then $g$ is realized by a vector-valued function $\mathbf{r}$ as in (2.1) if and only if (1.6) is satisfied.

Proof. The discussion before the statement of Lemma 2.2 shows that (1.6) is a necessary condition for $g$ to be realized by $\mathbf{r}$ as in (2.1). Now we prove it is also sufficient. Let $g$ be a metric on $\mathbb{T}^{2}$ as in (1.5) and satisfy (2.7) and (1.6). Set

$$
f(t)=\sqrt{E(t)}
$$

and

$$
h(t)= \begin{cases}\int_{t_{1}}^{t} \sqrt{1-\left(\frac{E^{\prime}}{2 \sqrt{E G}}\right)^{2}} \sqrt{G} d s & \text { for } t \in\left(t_{1}, t_{2}\right) \\ \int_{t}^{t_{1}+2 \pi} \sqrt{1-\left(\frac{E^{\prime}}{2 \sqrt{E G}}\right)^{2}} \sqrt{G} d s & \text { for } t \in\left(t_{2}, t_{1}+2 \pi\right) .\end{cases}
$$

Then $h\left(t_{2}-\right)=h\left(t_{2}+\right)$ by $(1.6)$ and hence $h$ is a well-defined function on $\mathbb{S}^{1}$. A straightforward calculation shows that $\mathbf{r}$ defined by (2.1) with such $f$ and $h$ is an isometric embedding of the metric $g$.

We point out that (1.6) is the extra condition we attempt to seek in order to solve Problem 1.1 for metrics as in (1.5). In fact, (1.6) has a significant geometric implication. As the proof of Lemma 2.2 shows, in order to construct a desired isometric embedding, we start from a circle $\left\{t=t_{1}\right\}$ where the Gauss curvature is zero and construct parts of the surface where the Gauss curvature is positive and negative separately. Then (1.6) implies that these two parts coincide at another circle $\left\{t=t_{2}\right\}$ where the Gauss curvature is zero to form a closed surface. If (1.6) is violated, then no closed surface of revolution realizes the metric.

In Lemma 2.2, the isometric embedding of $g$ is required to be a surface of revolution in $\mathbb{R}^{3}$. There is no such a requirement in Theorem 1.2. In a sense, Theorem 1.2 supplements Lemma 2.2 by adding the uniqueness. The proof of Theorem 1.2 is based on a rigidity result due to Nirenberg [14] which we now state.

THEOREM 2.3. Let $M$ be a smooth closed oriented surface in $\mathbb{R}^{3}$ satisfying (1.2) and (1.3). Then $M_{+}=\{p \in M ; K(p)>0\}$ is rigid. Moreover, if there is at most one closed asymptotic curve in each component of $M_{-}=\{p \in M ; K(p)<0\}$, then $M$ is rigid.

The assumption on closed asymptotic curves is non-intrinsic. It should be understood in the following way. Let $M^{\prime}$ be another smooth closed oriented surface in $\mathbb{R}^{3}$ isometric to $M$, then $M^{\prime}$ is congruent to $M$. We do not need to put the assumption on the closed asymptotic curves on $M^{\prime}$. The proof of the rigidity of $M_{-}$is based on the uniqueness of solutions of Gauss-Codazzi system. Asymptotic curves form characteristic curves for this system. The assumption on closed asymptotic curves enables 
characteristic curves to reach every point except a closed curve in each component of $M_{-}$. See Theorem 8.1.4 in [6] for details.

We are ready to prove Theorem 1.2.

Proof of Theorem 1.2. As in the proof of Lemma 2.2, we set $f=\sqrt{E}$ and construct $h$ from $t=t_{1}$. Geometrically, this defines the components $M_{+}=\{p \in M ; K(p)>0\}$ and $M_{-}=\{p \in M ; K(p)<0\}$. The rigidity of $M_{+}$follows from the first part of Theorem 2.3. In order to apply the second part of Theorem 2.3, we need to verify that there are no closed asymptotic curves on $M_{-}$. This is based on the fact that $M_{-}$ is a surface of revolution. With $\mathbf{r}$ given by (2.1), the second fundamental form $I I$ is given by (2.3), i.e.,

$$
I I=\frac{1}{f^{\prime 2}+g^{\prime 2}}\left(-f h^{\prime} d x^{2}+\left(h^{\prime} f^{\prime \prime}-f^{\prime} h^{\prime \prime}\right) d t^{2}\right) .
$$

Then any asymptotic curve $t=t(x)$ satisfies

$$
\left(\frac{d t}{d x}\right)^{2}=\frac{f h^{\prime}}{h^{\prime} f^{\prime \prime}-f^{\prime} h^{\prime \prime}} .
$$

By $K<0$ in $M_{-}$and $K=0, \nabla K \neq 0$ on $\partial M_{-}$, we have $f\left(h^{\prime} f^{\prime \prime}-f^{\prime} h^{\prime \prime}\right) \neq 0$ on $\bar{M}_{-}$, $h^{\prime} \neq 0$ on $M_{-}$and $h^{\prime}=0$ on $\partial M_{-}$. A simple argument shows that any asymptotic curves starting from $t=t_{1}$ will reach $t=t_{2}$. Therefore, there are no closed asymptotic curves in $M_{-}$. The proof of Theorem 2.3, implies that the Gauss-Codazzi system has a unique solution with the initial condition at $t=t_{1}$ induced from $M_{+}$. Hence, any isometric embedding of $\left(\mathbb{T}^{2}, g\right)$ is a surface of revolution in $\mathbb{R}^{3}$. Now we can apply Lemma 2.2 to yield the desired result.

With Theorem 1.2, we can construct metrics on torus which does not admit isometric embedding in $\mathbb{R}^{3}$ and these metrics are perturbations of the standard metric on torus. For example, we consider a family of smooth positive functions $E_{\lambda}$ on $\mathbb{S}^{1}$ such that

$$
E_{0}=(a+\cos t)^{2},
$$

for some constant $a>1$, and for $\lambda \neq 0$

$$
\begin{aligned}
& \left(\sqrt{E_{\lambda}}\right)^{\prime \prime}>0 \text { on }\left(t_{\lambda 1}, t_{\lambda 2}\right), \quad\left(\sqrt{E_{\lambda}}\right)^{\prime \prime}<0 \text { on }\left(t_{\lambda 2}, t_{\lambda_{1}}+2 \pi\right), \\
& \left|\left(\sqrt{E_{\lambda}}\right)^{\prime}\right| \leq 1 \text { on } \mathbb{S}^{1}, \text { and }\left(\sqrt{E_{\lambda}}\right)^{\prime}\left(t_{\lambda 1}\right)=1,\left(\sqrt{E_{\lambda}}\right)^{\prime}\left(t_{\lambda 2}\right)=-1, \\
& \int_{t_{\lambda 1}}^{t_{\lambda 2}} \sqrt{1-\left(\sqrt{E_{\lambda}}\right)^{\prime 2}} d t \neq \int_{t_{\lambda 2}}^{t_{\lambda 1}+2 \pi} \sqrt{1-\left(\sqrt{E_{\lambda}}\right)^{\prime 2}} d t
\end{aligned}
$$

for some $t_{\lambda 1}, t_{\lambda 2} \in[0,2 \pi)$ with $t_{\lambda 1}<t_{\lambda 2}$. It is easy to construct such a family of functions $\left\{E_{\lambda}\right\}$. In fact, $t_{\lambda 1}, t_{\lambda 2}$ can be chosen independently of $\lambda$. For example, we may take $t_{\lambda 1}=-\pi / 2, t_{\lambda 2}=\pi / 2$ and make small perturbations of $E_{0}$ in $(-\pi / 2, \pi / 2)$ and $(\pi / 2,3 \pi / 2)$ to satisfy the requirements. Then we set

$$
g_{\lambda}=E_{\lambda}(t) d x^{2}+d t^{2} .
$$

Note that $E_{\lambda}$ and $G=1$ satisfy (2.7), but not (1.6) for $\lambda \neq 0$. Hence Theorem 1.2 implies that $\left(\mathbb{T}^{2}, g_{\lambda}\right)$ does not admit a smooth isometric embedding in $\mathbb{R}^{3}$. 


\section{REFERENCES}

[1] A. D. Alexandroff, On a class of closed surfaces, Recueil Math. (Moscow), 4 (1938), pp. 6977.

[2] A. D. Alexandroff, Existence of a polyhedron and of a convex surface with given metric, Dokl. Akad. Nauk SSSR (N.S.), 50 (1941), pp. 103-106; Mat. Sbornic (N.S.), 11(53)(1942), pp. $15-65$.

[3] S. E. Cohn-Vossen, Zwei Sätze über die Starrheit der Eiflächen, Nach. Gesellschaft Wiss. Göttinger, Math. Phys. Kl., (1927), pp. 125-134.

[4] P. GuAn And Y. Li, The Weyl problem with nonnegative Gauss curvature, J. Diff. Geometry, 39 (1994), pp. 331-342.

[5] M. GüNTHER, Isometric embeddings of Riemannian manifolds, Proceeding of the International Congress of Mathematicians, Kyoto, Japan, 1990, pp. 1137-1143.

[6] Q. Han And J.-X. Hong, Isometric Embedding of Riemannian Manifolds in Euclidean Spaces, Mathematical Surveys and Monographs, Volume 130, American Mathematical Society, Providence, RI, 2006.

[7] E. Heinz, On Weyl's embedding problem, J. Math. Mech., 11 (1962), pp. 421-454.

[8] G. Herglotz, Über die Starrheit der Eiflächen, Abh. Math. Sem. Hansischen Univ., 15 (1943), pp. $127-129$.

[9] J.-X. Hong AND C. ZuiLy, Isometric embedding of the 2-sphere with nonnegative curvature in $\mathbb{R}^{3}$, Math. Z., 219 (1995), pp. 323-334.

[10] H. LEWY, On the existence of a closed convex surface realizing a given Riemannian metric, Proc. Nat. Acad. Sci., 24 (1938), pp. 104-106.

[11] C.-S. LIN, The local isometric embedding in $\mathbb{R}^{3}$ of two dimensional Riemannian manifolds with Gaussian curvature changing sign clearly, Comm. Pure Appl. Math., 39 (1986), pp. 867887.

[12] J. NAsh, The embedding problem for Riemannian manifolds, Ann. of Math., 63 (1956), pp. 2063.

[13] L. Nirenberg, The Weyl and Minkowski problems in differential geometry in the large, Comm. Pure Appl. Math., 6 (1953), pp. 337-394.

[14] L. Nirenberg, Rigidity of a class of closed surfaces, Nonlinear Problems, edited by R. Lang, pp. 177-193, University of Wisconsin Press, 1963.

[15] A. V. Pogorelov, Regularity of a convex surface with given Gaussian curvature, (Russian) Mat. Sbornik (N.S.), 31 (1952), pp. 88-103.

[16] H. WEYL, Über die Bestimmheit einer geschlossenen konvex Fläche durch ihr Linienelement, Vierteljahresschrift der nat.-Forsch. Ges. Zürich, 61 (1916), pp. 40-72.

[17] S.-T. YAu, Problem Section, Seminar on Differential Geometry, Annals of Math. Studies, 102, Princeton University Press, 1982. 